\title{
Conceptualización de las pautas de crianza de buen trato y maltrato infantil, en familias del estrato socio- económico bajo: Una mirada Cualitativa
}

\section{A Framework for Child Treatment and Mistreatment in Poor Families: A Qualitative View.}

\author{
Marcela Aracena* \\ Eliana Balladares ${ }^{* *}$ \\ Francisca Román ${ }^{* * *}$ \\ Carolina Weiss ${ }^{* * * *}$
}

\begin{abstract}
Resumen
El maltrato infantil es un fenómeno complejo y multifacético en el cual inciden variables del sistema familiar, así como del contexto social. Uno de los problemas centrales en la investigación acerca del maltrato infantil es la conceptualización del fenómeno. En este artículo se presentan y discuten los resultados de una investigación cualitativa acerca de la conceptualización del maltrato infantil. En una primera parte, se presenta una revisión de las principales dificultades para definir lo que se entiende por «maltrato infantil», que han sido reportadas en la literatura. Se analizan específicamente los siguientes aspectos: (a) dificultades metodológicas, (b) aspectos históricos y culturales, (c) posturas de distintas disciplinas e (d) implicancias en la medición de la magnitud del problema.

En la segunda parte, se presenta la metodología cualitativa aplicada, y los principales hallazgos de la investigación cuyo objetivo era conocer las pautas de crianza y la conceptualización del maltrato infantil en la ciudad de Temuco; percibida desde la perspectiva de profesionales y representantes de la comunidad.

Los principales hallazgos se presentan en cinco categorías: (a) pautas de crianza de buen trato, (b) valores predominantes, (c) maltrato físico, (d) maltrato emocional, y (e) abuso sexual.

Finalmente se discuten los hallazgos a la luz de los resultados de las investigaciones en el ámbito internacional y las implicancias en las políticas de intervención social.
\end{abstract}

Palabras claves: Conceptualización, Pautas de Buen trato, Maltrato infantil.

\footnotetext{
* Académico, Escuela de Psicología, Facultad de Ciencias Sociales, Pontificia Universidad Católica de Chile. Chile. e-mail:maracena@puc.cl

Esta investigación fue realizada gracias al apoyo financiero de la Fundación Rockefeller.

** Psicóloga, Departamento de Psicología de la Universidad de la Frontera. Chile. E-mail:gballad@ufro.cl

*** Psicóloga, Departamento de Psicología de la Universidad de La Frontera. Chile. E-mail: mroman@ufro.cl

***Psicóloga candidata a Magister de la Escuela de Psicología de la Pontificia Universidad Católica de Chile. Chile.

E-mail: cweiss@puc.cl
} 


\begin{abstract}
Child abuse is a complex and multidimensional phenomenon, which is influenced by variables from the family system and the social context. One of the central problems of research on child abuse is conceptualizing the phenomenon. This article presents and discusses results of a qualitative study on child abuse conceptualization. The first part includes a review of the main difficulties involved in defining what is meant by "child abuse" that have been reported in the literature. Specifically, the following issues are analyzed: (a) methodological difficulties, (b) historical and cultural issues, (c) viewpoints from different disciplines, and (d) implications for assessing the problem's magnitude.

The second part includes a description of the qualitative method used and the main findings of the study, which was targeted at inquiring about child rearing patterns and conceptualizations of child abuse in Temuco; as perceived from the perspective of professionals and community members. The main findings are presented in five categories: (a) child rearing patterns of good treatment, (b) prevailing values, (c) physical abuse, (d) emotional abuse, and (e) sexual abuse.

Lastly, the findings are discussed taking into account results from international research in the area and implications from social intervention policies.
\end{abstract}

Key words: Conception, good treatment patterns, child mishadle.

\section{Introducción}

La mayoría de los estudios realizados en maltrato infantil (M.I.) ${ }^{1}$ han sido dirigidos hacia la identificación de los factores asociados a este fenómeno y la epidemiología (prevalencia), y pocos estudios han centrado su interés en los problemas de conceptualización.

Un punto de partida fundamental para el estudio de un problema es definirlo. Sin embargo, la falta de claridad y ambigüedad en las definiciones ha sido la mayor dificultad en investigaciones sobre M.I. (Korbin, 1980, citado en Gelles \& Lancaster, 1987; Starr, Dubowitz \& Bush, 1990; Newberger, Moore \& Hamton, 1983; Howing, Wodarski, Kurtz \& Gaudin, 1989; Shaw, 1986; Haugaard \& Emery, 1989; Besharov, 1981; Gelles, 1987).
Existen muchas definiciones de M.I., ninguna de las cuales es mundialmente aceptada. Algunas explicaciones enfatizan que las definiciones son distintas porque las culturas difieren marcadamente unas de otras en: (a) las pautas de crianza, (b) el grado en que se reconoce a la infancia como una etapa del desarrollo que merece una atención especial, (c) las condiciones que se consideran necesarias para un desarrollo saludable, y (d) los derechos establecidos hacia los niños (Eisenberg, 1981).

El grado en que un país reconoce el M.I. depende de las definiciones locales, tradiciones y prioridades (Gelles, 1987). En suma, las diferencias en las definiciones pueden darse entre y al interior de las culturas. Estas definiciones pueden variar de acuerdo a las profesiones, investigadores, estados, organizaciones y también de acuerdo a los distintos niveles socioeconómicos

1 El concepto de maltrato infantil que se emplea en este artículo hace referencia las siguientes subcategorías: (a) maltrato físico, (b) maltrato psicológico, (c) abuso sexual, (d) negligencia física, y (e) negligencia psicológica. 
(Starr, Dubowitz \& Bush, 1990; Gelles, 1982; Valentine, Steward, Freeman \& Andreas, 1984; Biller \& Solomon, 1986).

En la literatura, se señala que el problema central para llegar a una definición histórica y transcultural de M.I., se basa en los valores de la sociedad en su totalidad (Gelles, 1987). Existen diferencias considerables en normas y valores entre las distintas sociedades y dentro de cada una de ellas, y a través del tiempo; siendo éstas las dificultades para establecer los límites entre una paternidad normal y abusiva (Gelles, 1987).

Como Kempe (1982) enfatiza, las definiciones de M.I. varían de una sociedad a otra y a través del tiempo; pudiendo reflejar esto las necesidades de supervivencia del grupo. Dentro de este contexto, Korbin (1980) señala que esta postura presenta un dilema cultural. La autora señala que si la sociedad no considera una perspectiva cultural en la definición de M.I. puede verse en una posición etnocéntrica, en la cual el propio sistema de creencias y prácticas culturales presupone que éstas son superiores y preferibles que otras definiciones. Al mismo tiempo, la autora argumenta que no es posible tomar una posición de extremo relativismo cultural, con relación a cómo se trata a los niños. Para conciliar este dilema, se sugiere que es necesario estructurar una definición conceptual de M.I. que permita identificar apropiadamente el fenómeno dentro y entre los contextos culturales y socioeconómicos.

Las dificultades encontradas en los estudios acerca de M.I. ponen de manifiesto la necesidad de contar con una definición clara de este concepto. Uno de los aspectos más difíciles de abordar en esta área, es determinar quién definirá «maltrato». Al respecto, se han desarrollado dos posturas durante las últimas tres décadas. La primera plantea que la definición debe estar elaborada por los profesionales; la segunda, propone que la definición debe ser el resultado de un proceso social en que los distintos sectores de la comunidad hayan sido consultados (Hutchison, 1990). Ambas posturas encuentran apoyo en la literatura; por lo que el dilema de quién define, aún no ha sido satisfactoriamente resuelto. A pesar de ello, la aproximación más utilizada a nivel internacional, es el punto de vista de los profesionales.

La definición de M.I. ha evolucionado a través de la historia. A partir de la revisión de la literatura, es posible argumentar que las definiciones son el resultado no sólo de un proceso de toma de conciencia acerca del fenómeno descrito por Kempe (1982); sino que responden a las diferentes perspectivas, dependiendo del observador y las bases teóricas en las cuales éste se sustenta. Con relación a la toma de conciencia respecto al maltrato, Kempe (1982) describe cinco etapas: negación del problema, reconocimiento de los casos de maltrato físico, eficacia del tratamiento del maltrato físico, reconocimiento del maltrato emocional y negligencia, y cuando hay reconocimiento del abuso sexual. Respecto a las diferentes perspectivas teóricas, existen cuatro aproximaciones a la definición de maltrato infantil: (a) perspectiva legal, (b) perspectiva médico-psicológica, (c) perspectiva de las ciencias sociales, y (d) perspectiva de la investigación.

Finalmente, la carencia de claridad y de acuerdos acerca de las definiciones de maltrato infantil que son utilizadas por los sistemas sociales, legales y de salud constituyen la principal dificultad metodológica en el estudio del problema, ya que los resultados que se obtienen en las investigaciones difieren significativamente y por ende, no permiten obtener conclusiones válidas y confiables que permitan explicar el maltrato infantil (Korbin, citado en Gelles \& Lancaster, 1987).

La falta de conceptualización y operacionalización clara acerca de M.I. limita el desarrollo de estrategias que permitan predecirlo (Gelles, 1982). De este modo, se dificulta la tarea de crear programas de in- 
tervención adecuados que posibiliten alcanzar el objetivo último de proteger al niño de la violencia (Valentine \& col., 1984).

Chile está dentro de los países en los cuales ha habido un interés creciente por el tema, como asimismo un reconocimiento gradual frente a este fenómeno en el último decenio (De la Barra \& col., 1991; Ley 19.225, Diario Oficial de la República de Chile, 27 de Agosto de 1994).

En este contexto la presente investigación descriptiva de carácter cualitativo tiene por objetivo: conocer las pautas de buen trato y la conceptualización de maltrato infantil en la ciudad de $\mathrm{Temuco}^{2}$; percibida desde la perspectiva de profesionales y representantes de la comunidad.

\section{Método}

\section{Diseño}

Se empleó un diseño descriptivo cualitativo que permitió explorar en forma directa las creencias y las opiniones de las personas en el grupo estudiado.

\section{Sujetos}

El estudio utilizó una muestra intencionada de 75 personas: 36 profesionales, 6 líderes de la comunidad y 33 padres y apoderados de la comunidad estudiada. Se empleó la estrategia de muestreo denominada Snowball para acceder a los participantes (Patton, 1990).

El criterio para elegir los profesionales fue la experiencia laboral en la problemática del maltrato infantil en el nivel socioeconómico bajo. Participan en esta investiga- ción 27 profesionales de sexo femenino y 9 profesionales varones con una edad promedio de 45 años de los campos de la salud, educación y justicia ${ }^{3}$.

Los líderes de la comunidad se eligen a través de informantes claves de instituciones formales de la comunidad. Este grupo queda constituido por 5 presidentas o vicepresidentes de Junta de Vecinos y un presidente de una Junta de Vecinos de sexo masculino. Las edades fluctuaban entre 35 y 45 años de edad.

Los padres y apoderados de la comunidad se eligen a través de informantes claves de establecimientos educacionales. Para ingresar a este grupo se solicita a los informantes invitar a padres o apoderados que se destaquen por "buenos padres" y no cuenten con antecedentes de maltrato infantil en servicios públicos. Participan en este grupo 23 madres y 10 padres de familia, con una edad promedio de 35 años.

\section{Técnica de recolección de información}

Se eligió la técnica de grupo focal para conocer la conceptualización de maltrato infantil. Se llevaron a cabo 19 sesiones de discusión con un total de 38 horas cronológicas.

\section{Procedimiento}

Los participantes fueron invitados a través de cartas personales enviadas a sus instituciones de trabajo. Los grupos focales se llevaron a cabo haciendo uso de una pauta de trabajo enviada con anterioridad a los participantes que incluía los siguientes tópicos: (a) pautas de crianza, (b) concepto de maltrato infantil, y (c) terminología (ver tabla 1).

2 Temuco es la capital de la IX Región de Chile

3 En el grupo de expertos participan profesionales de Carabineros de Chile, Policía de Investigaciones, Servicio de Pediatría, Servicio de Medicina Legal, Servicio de Urgencia Infantil, Centro de Salud Mental Familiar, Centro de Observación Tránsito y Diagnóstico, Juzgado de Menores, Centro de derechos de la Mujer, Servicio Nacional de la Mujer, Escuela de Psicología de la Universidad de La Frontera y Departamento Educacional Municipal. 


\section{Tabla 1.}

\section{Tópicos incluidos en la pauta de discusión utilizada en los grupos focales}

\begin{tabular}{|c|c|}
\hline \multirow{5}{*}{ Pautas de Crianza } & - Pautas de crianza utilizadas en la comunidad \\
\hline & $\begin{array}{l}\text { - Diferencias de pautas de crianza según nivel } \\
\text { socioeconómico. }\end{array}$ \\
\hline & - Criterios de elección de las pautas de crianza \\
\hline & $\begin{array}{l}\text { - Métodos empleados frente a conductas-problemas } \\
\text { de los niños }\end{array}$ \\
\hline & - Ejemplos \\
\hline \multirow{5}{*}{ Concepto de maltrato infantil } & - Definiciones \\
\hline & - Criterios operacionales para su definición \\
\hline & $\begin{array}{l}\text { - Características asociadas a las personas que } \\
\text { ejercen maltrato }\end{array}$ \\
\hline & $\begin{array}{l}\text { - Criterios empleados por la comunidad para } \\
\text { reconocer un caso de maltrato }\end{array}$ \\
\hline & - Ejemplos \\
\hline Terminología & $\begin{array}{l}\text { - Términos empleados por la comunidad para } \\
\text { denominar situaciones de maltrato físico y } \\
\text { emocional. }\end{array}$ \\
\hline
\end{tabular}

Todas las sesiones fueron grabadas con el consentimiento de los participantes. Dos ayudantes de investigación tomaron notas en forma independiente en cada uno de los grupos focales. Toda la información fue transcrita a documentos y analizada por el equipo de investigación paralelamente a la recolección de los datos. Además, se realizó un resumen de cada reunión de trabajo el cual fue entregado a cada participante para su aprobación o modificación, realizando una segunda versión del resumen cuando fue necesario.

En todo el proceso de recolección de la información se empleó la estrategia de triangulación. Se utilizó la estrategia de triangulación por observador y fuentes de información, con el objeto de asegurar la confiabilidad y validez de los resultados (Patton, 1990).

\section{Análisis e Interpretación}

Para analizar la conceptualización de maltrato infantil se aplicaron los procedimientos de la "Grounded Theory" de Glaser y Strauss (Strauss \& Corbin, 1990). Para tal efecto, el primer paso es la codificación de los datos obtenidos. Codificar significa extraer pasajes, conceptos o formulaciones claves que permiten articular analíticamente el material analizado (Krausse, 1995). Para efectos del presente estudio se utiliza la codificación abierta. La estrategia que se aplica a los datos codificados abiertamente es la "comparación permanente". Los resultados que se van generando a partir de estas comparaciones se registran en forma de memos, los cuales se van constituyendo en piezas de un modelo descriptivo acerca de la conceptualización de maltrato infantil. 
Para asegurar el nivel de confiabilidad y validez de los resultados, se realizó triangulación de investigadores durante el proceso de codificación e interpretación de la información.

\section{Resultados}

Los principales hallazgos referidos a la conceptualización de pautas de buen trato y maltrato intrafamiliar, se presentan en cinco categorías: (a) continuo de buen trato al maltrato, (b) valores predominantes, (c) maltrato físico, (d) maltrato emocional, y (e) abuso sexual. En cada una de las categorías de análisis se discuten aquellos temas que se identifican con mayor interés en el grupo estudiado.

\section{Continuo de Buen Trato y Maltrato}

Los participantes plantean que el concepto de pautas de crianza es complejo en su definición e incluye a lo menos cinco aspectos relevantes: (a) los valores de la sociedad, (b) una visión y relación con el mundo, (c) límites y normas sociales, (d) una apreciación de sí mismo y finalmente (e) una relación afectiva entre las partes.

La información recogida en esta investigación enfatiza que las pautas de crianza son válidas para un grupo social dependiendo de «quien» defina lo que es «normal» o «valorado». Los profesionales por ejemplo, señalan adscribirse a dos posturas diferentes dependiendo del rol que asumen, ya sea como padres o como profesionales. Ellos plantean que frecuentemente el patrón de comparación que utilizan es el personal, más que del grupo de referencia con que trabajan. Desde esta perspectiva, un análisis de las pautas de crianza utilizadas por un grupo determinado se complejiza debido a que cualquier observador cuenta con un marco de referencia personal con respecto a las pautas de crianza que son consideradas adecuadas o normales.

Se señala que las pautas de crianza en una sociedad se transmiten durante el proceso de desarrollo de un niño, este proceso se encuentra inserto en una relación afectiva entre un adulto y un menor. Se comenta que el proceso de crianza es gradual y que la familia no es siempre el agente de socialización más importante, sino en ocasiones la televisión, entre otros, pueden pasar a ocupar un rol central.

Durante las discusiones en los grupos focales surge el concepto de continuo "pautas de buen trato-maltrato». Cuando se habla de este concepto se quiere hacer referencia a aquel continuo en el cual, se pueden encontrar todas las estrategias que utiliza un adulto con el fin de transmitir a la próxima generación aquellos valores, pautas y creencias acerca de su propia comunidad. Es importante señalar que en un extremo se ubican aquellas estrategias que por consenso social, son adecuadas para transmitir lo "valorado" y en el otro extremo, se encuentran aquellas estrategias que la sociedad rechaza como medios para alcanzar el mismo fin (ver Figura 1). 
Figura 1

\section{Criterios para Distinguir cuando una conducta de Crianza se Transforma en} Maltrato Infantil

\begin{tabular}{|c|c|c|c|}
\hline & AREA DE DESACUERDO & & \\
\hline & Valores Culturales & & \\
\hline & Etapa de Desarrollo & & \\
\hline & Contexto y significado & & \\
\hline & Relación entre falta y & & \\
\hline & conducta del adulto & & \\
\hline & Control Emocional & & \\
\hline & Intensidad & & \\
\hline & Frecuencia & & \\
\hline $\begin{array}{l}\text { CONDUCTAS Recompensa } \\
\text { DE CRIANZA } \\
\text { SOCIALMENTE }\end{array}$ & $\begin{array}{ll}\text { Amenaza } & \begin{array}{l}\text { Castigo físico sin } \\
\text { lesión evidente }\end{array}\end{array}$ & $\begin{array}{l}\text { Golpe con } \\
\text { lesión evidente }\end{array}$ & $\begin{array}{l}\text { MALTRATO } \\
\text { INFANTIL }\end{array}$ \\
\hline
\end{tabular}

El que una estrategia sea utilizada como una pauta de buen trato, depende de una serie de factores, entre éstos se mencionan: (a) estado emocional de los padres, (b) nivel de tensión familiar, (c) expectativas de los padres, (d) nivel educacional, (e) dificultades económicas, e (f) historia de vida de los padres, entre otros.

El concepto de continuo en el campo del maltrato infantil sintetiza entonces la valoración que un determinado adulto realiza acerca de las estrategias empleadas en el proceso de socialización. Se encuentra por tanto que una misma estrategia puede ser percibida por diferentes adultos como apropiada o, no de acuerdo a los distintos niveles socioculturales.

Es por ello que este continuo puede responder a preguntas claves de por qué algunas conductas son percibidas como pautas de buen trato en un grupo determinado, en tanto que son percibidas como maltrato, en otros grupos socioculturales. La franja intermedia es el área que presenta mayores dificultades para su definición (ver Figura 1).

Existen criterios comunes que aparecen en los distintos grupos focales que permiten definir un comportamiento como maltrato infantil. Se conceptualiza un comportamiento como maltrato cuando existe huella física en el niño como consecuencia de la acción del adulto. La frecuencia y grado de control emocional del adulto son criterios adicionales que permiten ubicar una estrategia como maltrato infantil. Es decir, los participantes plantean que no hay duda que se trata de un caso de maltrato cuando el niño presenta una huella física como consecuencia del maltrato, cuando esta estrategia se utiliza en forma continua y reiterada -independiente de la conducta del niño- y finalmente cuando junto con lo anterior, se cuenta con antecedentes que, el adulto presenta dificultades para controlar sus impulsos frente a las conductas-problema del niño. 
Los criterios antes mencionados no permiten explicar la multiplicidad de combinaciones posibles. Variables como el contexto y la relación con el niño son algunos de los criterios que dificulta describir una conducta como pauta de buen trato en vez de maltrato infantil. Es por ello que resulta relevante poder describir en qué criterios la comunidad presenta consenso con los profesionales y en qué otros presenta divergencias, como asimismo, describir cómo el continuo descrito en el grupo estudiado puede diferir de otras realidades culturales.

A continuación se presentan algunos ejemplos de diferentes estrategias de crianza. En la muestra escogida se identifican 24 conductas. Las más comunes son aprobación/desaprobación, normas claras, recompensas, amenazas y castigo. Dentro de estas conductas, las amenazas, las recriminaciones verbales y el castigo físico de magnitud moderada (sin lesiones físicas) son estrategias en las cuales no hay consenso en los grupos focales. Estos últimos comportamientos son considerados maltrato de acuerdo a la perspectiva de los profesionales, no así desde la perspectiva de la comunidad.

\section{Valores predominantes}

Por otra parte, los participantes plantean la existencia de algunos valores predominantes en la comunidad estudiada que sustentan la conducta de maltrato infantil y que se transmiten a los niños en el proceso de crianza.

Entre los valores mencionados, los más significativos son: (a) individualismo, (b) competitividad, (c) machismo, y (d) valoración positiva de la violencia.

El valor del individualismo es ejemplificado por un profesional con el siguiente comentario: «la sociedad está demandando individuos más egocéntricos sobre la base de sus necesidades y en el no respeto por los otros»...»el individualismo y el egocentrismo traen como consecuencia la violencia».

Con respecto a la competitividad, se plantea en uno de los grupos focales que: «en este momento la sociedad exige ser competitivo ... Competitivo porque si uno se queda ahí, otro te come independiente de tus conocimientos, o sea la cosa es llegar primero, no importa como». La necesidad de sobrepasar a los demás constituye, otro de los valores generadores de violencia.

En relación con el machismo, se plantea que si bien éste está presente en toda la sociedad, parece ser más preponderante en el nivel socioeconómico bajo («en las clases bajas el hombre es el rey»). Es así como, la adhesión a los valores, creencias y conductas propias del machismo facilitan en la familia la aparición de violencia hacia la mujer y los niños.

Relacionado con lo anterior, en las discusiones se señala que la violencia es valorada positivamente al interior de la sociedad. Esto puede ser ejemplificado analizando la forma como se cría a los niños. Según la opinión de un líder comunitario: «el maltrato hacia los niños es una cuestión absolutamente normal en nuestra sociedad».

Se puede señalar que estos valores predominantes permiten comprender la forma como los distintos tipos de maltrato surgen en la comunidad estudiada, particularmente al interior de la familia.

\section{Conceptualización de maltrato infantil}

Según los profesionales, la conceptualización del maltrato infantil, se encuentra en una etapa de transición. Por una parte, el fenómeno está suscitando interés en los profesionales y autoridades. Se plantea que éste ha comenzado a convertirse en un fenómeno social desde el inicio del gobierno 
democrático. Se enfatiza que la apertura de los problemas de derechos humanos, junto con el compromiso del gobierno chileno de firmar el Acta de los Derechos del Niño, ha permitido que en todos los ámbitos sociales el tema hubiera sido válido para su discusión e intervención. Por otra parte, el maltrato, como una forma de relación, es considerado normal en la comunidad estudiada.

Al respecto, se plantea la dificultad para definir el concepto de maltrato infantil pese que a diario se encuentran en el trabajo con niños que viven experiencias de esta naturaleza. Sin embargo, se asegura que sólo se conoce una parte de todos los casos existentes, haciendo mención de una cifra negra de todos aquellos casos de maltrato infantil que no salen a la luz pública o que no llegan al sistema de salud o al sistema judicial.

Los participantes reconocen que el maltrato infantil es un problema social que se manifiesta en diversos niveles al interior de la sociedad. Se mencionan los siguientes: (a) familiar, (b) institucional, y (c) social.

\section{Criterios para una definición de maltrato físico 4}

Los participantes plantean algunos criterios que permiten establecer cuando una conducta puede ser denominada maltrato. Estos criterios son: (a) frecuencia, (b) intensidad, (c) grado de control emocional del adulto, (d) relación entre la falta y conducta emitida por el adulto, (e) contexto y significado para el niño, (f) etapa de desarrollo psicológico, cognitivo y físico, y (g) creencias culturales (ver Figura 1).
La intencionalidad y la conciencia con que se realiza la acción son discutidas como criterios adicionales para distinguir la conducta de maltrato, ya que no necesariamente están presentes al realizar esta conducta. No obstante, frente a estos dos últimos criterios, no existe consenso entre los participantes. Una de las posturas sostiene que la conducta de maltrato requiere ser intencional y consciente. Por otra parte, se plantea que independiente del grado de intencionalidad y conciencia, las consecuencias del maltrato igualmente están presentes en el niño.

Además, se sostiene que el maltrato físico puede o no dejar huellas corporales. En el caso que existan lesiones, el criterio para determinarlo sería: criterio médico (examen físico y de laboratorio). Cuando no hay lesiones, los participantes plantean que se podría hablar de maltrato, poniendo el énfasis en la conducta del agresor. Dentro de esta última posición, la conducta de maltrato puede ser considerada como la culminación de un proceso de interacción entre el guardador y el niño.

Se mencionan treinta y cuatro conductas con los cuales se denomina al maltrato físico. Estos se pueden agrupar en nueve categorías: (a) lanzar un objeto al niño; (b) apretar o sacudir con violencia (ejemplos: tirar la parte de la cara que más duele, tirar el pelo, tirar las orejas o patillas, dar un pellizcón, sacudir y zamarrear ${ }^{5}$ ); (c) abofetear (ejemplos: dar palmazos o palmadas, cachetadas, coscacho, coscorrón, charchazo); (d) dar puntapiés; (e) herir o tratar de herir con algo (ejemplos: chicotear, guasquear, pegar con la correa, cinturón o palo, colgar de extremidades, ama-

4 Los participantes sugieren que se reemplace el término abuso físico, el que es utilizado en la literatura internacional, por maltrato físico, ya que esta última es una expresión más común al interior de la comunidad. 5 El término "Zamarrear" se asemeja a lo descrito en la literatura como una de las manifestaciones del sindrome del niño sacudido. 
rrar las manos o el niño a la cama, sumergir en agua, duchas frías o baños de agua fría); (f) dar una paliza o golpiza (ejemplos: "dar la tanda", "dar la tunda", "dar la torta", "dar la calda"); y (g) quemar (ejemplos: tirar agua caliente, quemar con cigarrillos).

Con respecto a las consecuencias físicas del maltrato, se plantea que éstas pueden ser de dos tipos: (a) producir dolor y (b) producir daño físico o dejar huellas corporales (por ejemplo: hematomas, "cabeza rota», cojera, dolores en el cuerpo, dificultad para mover extremidades, entre otros). Sin embargo, se plantea que en algunas ocasiones no necesariamente quedan huellas corporales como consecuencia del maltrato físico.

Por otra parte, se plantea que este tipo de maltrato acarrea consecuencias psicológicas tales como: (a) baja autoestima, (b) retraimiento, (c) agresividad, (d) timidez, (e) dificultad para estudiar, (f) inquietud, y (g) desesperanza. Algunas consecuencias psicológicas del maltrato físico las ejemplifica un participante con la siguiente afirmación: «al niño lo van matando día a día».

\section{Criterios para una definición de maltra- to emocional ${ }^{6}$}

Con respecto a los criterios que permiten denominar a una conducta como maltrato emocional, los participantes mencionan los siguientes: (a) sensibilidad del niño frente a la acción del adulto (una misma acción puede tener efectos diferentes de acuerdo a las características individuales del niño, específicamente su grado de receptividad frente a la acción del adulto), (b) frecuencia de la acción, (c) edad del niño, y (d) consecuencias que se pueden observar en el niño (disminución de la autoestima, trastornos conductuales, agresividad, dificultad para desenvolverse en el medio, pasividad extrema, y dificultades de aprendizaje entre otras).

Al igual que en el maltrato físico, la intencionalidad es un elemento que puede o no estar presente en la conducta de maltrato emocional. Al respecto, se plantea que su objetivo puede ser controlar la conducta del niño y/o herir o causar daño.

Se mencionan veinte conductas con los cuales se denomina el maltrato emocional, los cuales se pueden agrupar en tres categorías: (a) gritar; (b) hacer o decir algo para herir al niño; y (c) amenazar (con pegar, con lanzar agua, entre otras) ${ }^{7}$.

Con respecto a las consecuencias del maltrato emocional, se plantea la dificultad de establecer una relación causal entre maltrato emocional y consecuencias observables en el niño, a diferencia del maltrato físico. Esta situación obstaculiza el diagnóstico y denuncia del maltrato emocional.

Entre las consecuencias del maltrato emocional se mencionan: (a) disminución de funciones en el niño, no favoreciendo un desarrollo armónico, por ejemplo, disminución de la autoestima, (b) trastornos conductuales, (c) agresividad, (d) dificultad para desenvolverse en el medio, (e)

6 Al igual que para maltrato físico, los participantes sugieren que se reemplace el término abuso emocional, el que es utilizado en la literatura internacional, por maltrato emocional.

7 En esta categoría se mencionan una gran cantidad de ejemplos, entre los cuales se pueden citar los siguientes: poner sobrenombres, insultar, asustar con «el cuco» 0 «el hombre del saco», avergonzar, desconfirmar («no pescar»), privar de afecto, exponer al niño a presenciar peleas entre los padres, encerrar al niño(a), dejarlo sin comer, echarlo de la casa por la noche, negar permisos, desvalorizar, mandarlo a acostar, mantenerlo acostado por largo tiempo, quitar la televisión, despersonalizar al niño, menoscabar y decir frases como: "no te quiero», "te tengo odio», "me tenís aburría», «eres muy tonto», «tú estorbas», «déjame tranquilo», «no molestes», «nadie te quiere» y "cállate». 
pasividad extrema, (f) dificultades de aprendizaje, (g) rebeldía, (h) bajo rendimiento escolar, y (i) aprendizaje del modelo de violencia.

\section{Criterios para una definición de abuso sexual}

Según los participantes, el concepto de abuso sexual abarca la gama que va desde las insinuaciones sexuales hasta relaciones sexuales con el menor.

Los criterios para distinguir cuáles conductas son consideradas abuso sexual son las siguientes: (a) uso del menor por un adulto como objeto sexual, (b) desconocimiento o incapacidad del niño para darse cuenta que es un abuso, (c) intencionalidad sexual por parte del adulto (los participantes consideran que en este tipo de abuso la intencionalidad sería un elemento central), (d) excitación sexual en el adulto y en el niño, (e) contexto en que se da la acción, (f) persona que la realiza y su relación con el niño y (g) zonas del cuerpo involucradas (genitales $\mathrm{v} / \mathrm{s}$ otras zonas).

Con respecto al abuso sexual, se plantea que existe una gran variabilidad cultural en lo que respecta al contacto físico aceptado. Debido a esto, las conductas que son definidas como abuso sexual pueden variar entre una cultura y otra. En la comunidad estudiada, se plantea que es normal la expresión física de cariño hacia los niños de parte de sus padres.

Se mencionan algunos nombres para denominar al abuso sexual, los cuales pueden ser agrupados en seis categorías: (a) exposición a videos pornográficos (en la medida que el niño sea obligado a observarlos), (b) exhibicionismo, (c) abusos deshonestos (ejemplos: "toqueteos", "manoseos"), (d) penetración sexual, (e) incesto («mi papá me tomó») y (f) violación ("se aprovechó», intentó violarla, «la quería de mujer»).

\section{Discusión}

El presente estudio significa un avance en Chile con respecto a la conceptualización del maltrato infantil, considerando que un aspecto central en el abordaje de un problema es la definición que se haga de éste.

El grado en que Chile ha reconocido el fenómeno del maltrato infantil depende, como lo señala Gelles (1987), de las tradiciones, prioridades y definiciones locales. Esto se ve reflejado en las opiniones vertidas por los participantes, los cuales señalan como un hito relevante para el país la Declaración de los Derechos del Niño (Fondo de las Naciones Unidas para la Infancia1989) junto con un reconocimiento gradual en el último decenio de esta problemática. (Aracena, Román, Haz, Muñoz \& Bustos 1999).

Tal como Eisenberg (1981) ha planteado, no existe una definición de maltrato infantil aceptada universalmente, lo que coincide con la postura de los participantes de este estudio.

Por otra parte, distintos autores plantean que las definiciones pueden ser diferentes al interior de las culturas y particularmente pueden variar de acuerdo a los distintos niveles socioeconómicos, aspecto que también es avalado en esta investigación (Starr \& col., 1990; Gelles 1982; Valentine \& col., 1984; Biller \& Solomon, 1986).

Para explicar las razones que están detrás de estas diferencias, en la literatura se plantean tres tipos de fundamentaciones teóricas.

En primer lugar, las diferencias encontradas en las definiciones de maltrato en los distintos niveles socioeconómicos pueden deberse a las diferencias de normas y valores al interior de una sociedad, tal como Gelles (1987) lo ha señalado. Esto, explica las diferencias en los grupos estudiados en términos de establecer los límites entre la 
paternidad abusiva y normal, lo que se refleja en el continuo pautas de buen tratomaltrato, construido a partir de las opiniones de los participantes en los grupos focales.

En segundo lugar, Mash y Wolfe (1991) plantean que es necesario conceptualizar el maltrato infantil no sólo como conductas y acciones visibles por parte de un observador externo, sino que se hace necesario considerar en la definición de maltrato, la calidad general del cuidado que recibe el niño. Esta consideración permite incluir todos los aspectos que aparecen riesgosos para éste, lo que genera una definición más amplia que las que sólo consideran un tipo de comportamiento. Por ejemplo, considerar maltrato físico sólo cuando existe daño físico visible.

En tercer lugar, con respecto a la conceptualización del maltrato infantil, es importante destacar los planteamientos de Kempe (1978), relacionados con las etapas de reconocimiento social del maltrato infantil, y la forma en que esto se relaciona con la definición de maltrato. Este autor señala que existen diferentes etapas de reconocimiento del maltrato. Las sociedades estarían dispuestas a reconocer el tema del maltrato físico, posteriormente el maltrato emocional y el reconocimiento del abuso sexual es la última etapa en la toma de conciencia social del fenómeno del maltrato infantil.

En la comunidad estudiada, resulta más simple poder caracterizar y definir el maltrato físico que el maltrato emocional, ya que este último abarca una gama de conductas más amplia y diversa, arraigada en las creencias y valores de cada grupo.

Si bien el maltrato infantil es reconocido como un problema social relevante, los participantes reconocen que muchas de las creencias y prácticas acerca del maltrato infantil son percibidas por la comunidad como pautas de buen trato, más que mal- trato infantil. El maltrato es percibido como «algo normal» en la vida cotidiana de la comunidad. Existe claridad al nivel de ésta, que el fenómeno del maltrato está presente en el medio social y que el "castigo físico» es aceptado como pauta de crianza habitual.

Por otra parte, para los participantes, el abuso sexual aparece como un fenómeno que genera preocupación social, pero no con la misma fuerza, intensidad y frecuencia con que se plantea acerca del maltrato físico y emocional. Para el grupo estudiado, el que un adulto toque la espalda o las manos de un niño no es expresión de abuso sexual. No obstante, como plantea Barudy (1999), en un contexto abusivo, la seducción del abusador hacia el niño puede comenzar por expresiones físicas de esta naturaleza.

Sin embargo, pese a las diferencias entre maltrato físico, emocional y sexual y sus explicaciones, existen ciertos criterios entregados por los participantes, que permiten resolver el dilema planteado por Korbin (1980), acerca de la necesidad de contar con una definición culturalmente pertinente de maltrato infantil. Criterios como frecuencia, intensidad, control emocional y la relación entre la falta del niño y la conducta del adulto aparecen como elementos consensuales que permitirían la posibilidad de elaborar una definición de maltrato infantil de carácter más universal. No obstante, criterios como la intencionalidad en la respuesta del adulto y la receptividad del niño a las conductas del adulto; son temas sobre los que no existe acuerdo entre los participantes, en relación con su importancia a la hora de definir una conducta maltratadora.

Desde el punto de vista de las autoras resulta preocupante que la responsabilidad de delimitar si una conducta es o no maltrato, quede en manos del niño y no en las del adulto. 
Tal como lo ha planteado Korbin, es necesario que la sociedad no solamente contemple una perspectiva cultural en la definición conceptual que permita identificar un fenómeno dentro y entre los contextos culturales.

$\mathrm{Al}$ respecto, resulta especialmente esclarecedor el concepto de continuo «pautas de buen trato-maltrato», planteado en este estudio. A partir de este concepto se pueden clasificar distintas estrategias de crianza de acuerdo a una serie de criterios antes mencionados. Para cada grupo social o para cada cultura se pueden construir distintos continuos y compararse entre sí. Particularmente, en la comunidad estudiada este continuo permite comprender como en los diversos niveles socioeconómicos una misma conducta puede ser vista como pauta de buen trato o maltrato.

Por otra parte, Mash y Wolfe (1991) señalan que el maltrato físico rara vez ocurre en forma aislada de otros tipos de maltrato (maltrato emocional y negligencia, entre otros). Por tanto, realizar definiciones específicas y no contextualizadas limita los alcances de las conclusiones e impide avanzar en la comprensión del fenómeno.

A la fecha, en el área de investigación se ha estudiado el maltrato infantil con definiciones operacionales restrictivas, en las cuales se destaca la presencia de daño demostrable como criterio esencial para definir un caso de maltrato infantil (Aracena 1997, Haz 1998). En este tipo de definiciones han quedado excluidos una serie de consecuencias no visibles en el niño (baja autoestima, timidez y retraimiento, entre otras) que también pueden ser el resultado del maltrato (Barudy, 1999). Si bien lo anterior se ha justificado con criterios de objetividad científica (comparaciones entre grupos), paradojalmente no ha aumentado la posibilidad de establecer comparaciones entre distintos estudios, porque caracterizar maltrato físico sólo por una consecuencia -por ejemplo daño visible-, no permite captar otras dimensiones del fenómeno. Es por ello que conceptualizar en un continuo la relación entre buen trato y maltrato ofrece una posibilidad de operacionalizar un concepto difícil de aprehender, como lo que se entiende por maltrato infantil. Lo anterior cobra mayor relevancia al momento de determinar distintos puntos de corte dentro del continuo, que permitan diseñar estrategias de intervención más apropiadas.

Conceptualizar en un continuo, las conductas de buen trato-maltrato es una necesidad no sólo para aquellos que trabajan en investigación sino preferentemente para aquellos actores que están centrados en la prevención y tratamiento de las consecuencias del maltrato infantil.

En este continuo se pueden distinguir tres franjas, cada una de ellas compuesta por una serie de comportamientos que pueden ser catalogados como maltrato, riesgo de maltrato o buen trato. Cada uno de estos comportamientos entraría en interjuego con criterios como frecuencia, intensidad y contexto familiar entre otros, y a partir de esta interacción se produce el movimiento dentro del continuo, y por tanto, la denominación que se haga de estos comportamientos, ya sea como una conducta de buen trato, de riesgo o maltrato. Se sitúan en la franja de mayor severidad los comportamientos denominados como maltrato físico infantil. En la franja intermedia se ubica aquel grupo de conductas de riesgo que facilita la aparición de potenciales comportamientos maltratadores. Finalmente, se encuentra una franja en la cual se ubicaría un conjunto de comportamientos reconocidos por la comunidad como pautas de buen trato.

Considerando lo anterior, el tipo de intervención psicosocial indicada, varía según 
el punto del continuo donde el operador social $^{9}$ se sitúe. Por ejemplo, en el extremo del maltrato, sería recomendable un tratamiento de rehabilitación, centrado en acompañar al grupo familiar en el proceso de reconocimiento de su involucración en la dinámica de violencia. En la franja intermedia, corresponde aplicar medidas de prevención que permitan detectar indicadores de riesgo que faciliten la emergencia de ciclos de la violencia, tales como frecuencia, intensidad y contexto, entre otros. En el extremo más cercano al buen trato, las intervenciones deben ir en la línea de la promoción de la salud, por ejemplo a través de la implementación de técnicas de resolución de conflictos en forma no violenta, comunicación interpersonal, redes sociales, entre otros.

Una de las implicancias de los resultados de esta investigación en el diseño de políticas sociales, es la necesidad de reconocer que el maltrato es una realidad ya asentada en la vida cotidiana en nuestro país, que se expresa en comportamientos diversos, que tienen que ser definidos no sólo por una conducta aislada, por ejemplo daño físico visible, sino por una constelación de criterios que constituyan un comportamiento maltratador, tal como lo señalamos previamente. Por tanto, no son necesarios sólo programas preventivos y curativos; sino también políticas dirigidas a crear conciencia de cómo los valores y creencias arraigados en la comunidad estudiada, sustentan el fenómeno del maltrato infantil.

Por último, es necesario resaltar entonces, que cualquier intervención que se realice, ya sea en el ámbito primario, secundario o terciario, debe considerar estos aspectos culturales e idiosincrásicos de la población local.
Una pregunta importante a ser resuelta en futuras investigaciones es de qué manera la falta de consideración de aspectos culturales influye en la capacidad de los profesionales de la salud para realizar intervenciones efectivas en el ámbito de esta problemática.

\section{Referencias Bibliográficas}

Aracena, M., Román, F., Haz, A.M., Muñoz, S. \& Bustos, L. (1999). Pesquisa de maltrato infantil: Una dificultad metodológica o conceptual. Apreciaciones de investigaciones chilenas. Psykhe, $\underline{8}$ (1), 117-124.

Arón, A. M. (2001). Violencia en la Familia. Programa de intervención en red: La experiencia de San Bernardo. Santiago de Chile: Galdoc.

Barudy, J (1999). Maltrato Infantil. Ecología Social: Prevención y Reparación. Santiago de Chile: Galdoc.

Besharov, D. J. (1981). Toward better research on child abuse and neglect: making definitional issues and explicit methodological concern. Child Abuse $\mathcal{E}$ Neglect, 5, 383-390.

Biller, H. B., \& Solomon, R. S. (1986). Child maltreatment: The definitional dilemma. En H. B. Biller \& R. S. Solomon (Eds.), Child maltreatment and paternal deprivation. A manifesto for research, prevention, and treatment (pp. 9-20). Lexington, MA: D.C. Heath and Company.

De la Barra, F., Alvarez, P., Gilchrist, A., Alvarez, N., Gamboa, R, \& Novoa, F., (1991). Maltrato infantil en Chile, Santiago: Ministerio de Salud.

\footnotetext{
9 De acuerdo con la definición de operador social de Arón (2001), pueden ser operadores sociales en una intervención en crisis de primer orden: los padres, policías, el clero, educadores, abogados, médicos, enfermeras, trabajadores sociales y monitores entre otros.
} 
EisenberG, L. (1981). Cross-cultural and historical perspectives on child abuse and neglect. Child Abuse \& Neglect, 5, 299-308.

Fondo de las Naciones Unidas Para la ProTECCIÓN DE LA INFANCIA (1989). Convención de los Derechos del Niño. Santiago: UNICEF.

Gelles, R. J. (1982). Problems in defining and labeling child abuse. En R. H. Starr (Ed.), Child abuse prediction. Policy implications (pp. 1-30). Cambridge, MA: Ballinger Publishing.

Gelles, R. J. (1987). What to learn from cross-cultural and historical research on child abuse and neglect: an overview. In R. J. Gelles \& J. B. Lancaster (Eds.), Child abuse and neglect. Biosocial dimensions (pp. 15-30). New York: Aldine de Gruyter.

Gelles, R. J. \& Lancaster, J. B. (1987). Child abuse and neglect. Biosocial dimensions. New York: Aldine de Gruyter.

Haugaard, J. J. \& Emery, R. E. (1989). Methodological issues in child sexual abuse research. Child Abuse \& Neglect, 13, 89-100.

Haz, A. M. \& Ramírez, V. (1998). Preliminary validation of the child abuse potencial inventory in Chile. Child Abuse \& Neglect, 22 (9), 869-879.

Howing P. T., Wodarski, J. S., Kurtz, P. D. \& Gaudin, J. M. (1989). Methodological issues in child maltreatment research. Social Work Research \& Abstracts Sep:3-7.

Hutchison, E. D. (1990). Child maltreatment: can it be defined? Social Service Review, March, 60-78.

Kempe, C. H. (1978). Recent developments in the field of child abuse. Child Abuse $\mathcal{E}$ Neglect, 2, 261-267.
Kempe, C. H. (1982). Cross-cultural perspectives in child abuse. Pediatrics, 69, 497-498.

Korbin, J.E. (1980). The cultural context of child abuse and neglect. Child Abuse $\mathcal{E}$ Neglect, 4, 3-13.

KRAUSE, M. (1995). La investigación cualitativa: Un campo de posibilidades y desafios. Revista Temas de Educación. 7, 1-22.

Mash, J.E., \& Wolfe A. D. (1991). Methodological issues in research on physical child abuse. Criminal justice and behavior, 18, 8-29.

Newberger, E. H., Moore, C., Hampton, R. L. (1983). Child abuse: the current theory base and future research needs. Journal of the American Academy of Child Psychiatry, 22, 262-268.

Patton, M. Q. (1990). Qualitative evaluation and research methods (2 edición). Newbury Park, CA: Sage.

SHAW, V. (1986). The psychosocial adjustment of maltreated children: methodological limitations and guidelines for future research. Child Abuse \& Neglect, 10, 63-69.

Starr, R. H., Dubowitz, J., \& Bush, B. A. (1990). The epidemiology of child maltreatment. In R. T. Ammerman, \& M. Hersen (Eds.), Children at risk. An evaluation of factors contributing to child abuse and neglect (pp. 23-50). New York: Plenum Press.

Valentine, D. P., Stewart, D., Freeman, M. L., \& Andreas, T. (1984). Defining child maltreatment: a multidisciplinary overview. Child Welfare, 43, 497-509.

Wolfe, D. (1985). Child-abusive parents: An empirical review and analysis. Psychological Bulletin, 97, 462-482.

Período de límite de recepción de artículos: 30 de Marzo 2002.

Fecha de aceptación artículos: 30 de octubre 2002. 
